\title{
CINEMATICS OF THE 3R DYAD
}

Florian Ion PETRESCU

Relly Victoria PETRESCU,

\begin{abstract}
This paper presents some original methods to determine the kinematic parameters at the $3 R$ dyad. It is starting with a trigonometric method, which has the advantage to determine very quickly the positions angles. The velocities can be determined faster using a way vector, so, for the second (proposed) cinematic method, one uses the first trigonometric way for the positions determination, and the vector method for the determining of the velocities and the accelerations. The third proposed method, is a geometric method, which let us to determine some intermediate kinematic parameters (of the internal couple $C$ ), and then we can determine the main kinematic parameters (the rotation angles with their derivatives).
\end{abstract}

Keywords: 3R dyad, kinematic parameters, cinematic

1 Senior Lecturer Ph.D. Eng. - Bucharest Polytechnic University - Email: petrescuflorian@yahoo.com

2 Senior Lecturer Ph.D. Eng. - Bucharest Polytechnic University - Email: petrescuvictoria@yahoo.com 


\section{Introduction}

In this paper it presents three methods able to determine the kinematic parameters to a $3 \mathrm{R}$ dyad (see the Figure 1).

It is starting with a trigonometric method, which has the advantage to determine very quickly the positions angles [1-4].

The velocities can be determined faster using a way vector, so, for the second (proposed) cinematic method, one uses the first trigonometric way for the positions determination, and the vector method for the determining of the velocities and the accelerations [1], [4].

The third proposed method, is a geometric method, which let us to determine some intermediate kinematic parameters (of the internal couple C), and then we can determine the main kinematic parameters (the rotation angles with their derivatives) [4].

\section{A trigonometric method}

The kinematic schema of a RRR dyad can be seen in the Figure 1 [4].

Fig. 1. Kinematic schema of a $3 R$ Dyad

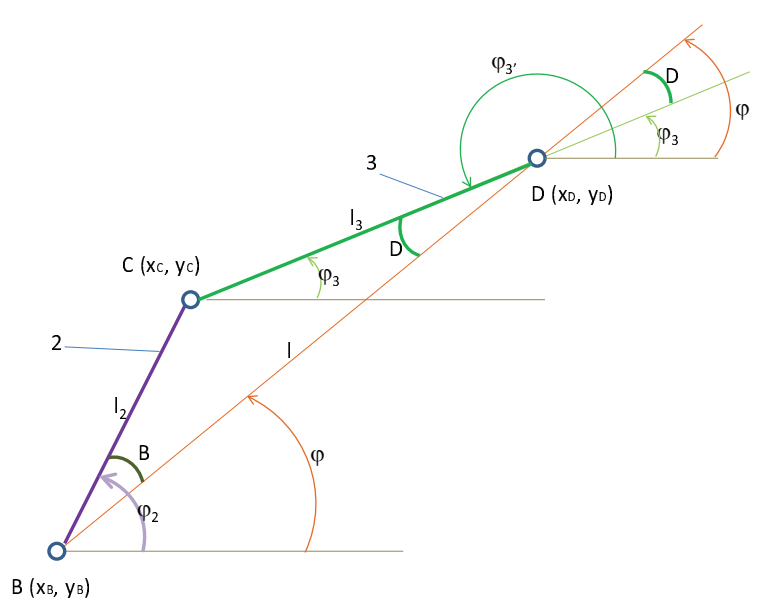

Following cinematic parameters are considered known (input):

$$
x_{B} ; y_{B} ; x_{D} ; y_{D} ; \dot{x}_{B} ; \dot{y}_{B} ; \dot{x}_{D} ; \dot{y}_{D} ; \ddot{x}_{B} ; \ddot{y}_{B} ; \ddot{x}_{D} ; \ddot{y}_{D}
$$

The question is to determine the next cinematic parameters (output):

$$
\varphi_{2}, \dot{\varphi}_{2} \equiv \omega_{2}, \ddot{\varphi}_{2} \equiv \varepsilon_{2}, \varphi_{3}, \dot{\varphi}_{3} \equiv \omega_{3}, \ddot{\varphi}_{3} \equiv \varepsilon_{3}
$$

It determines the angle $\varphi_{2}$ and then the angle $\varphi_{3}$ in function of the three angles: $\varphi, \hat{B}, \hat{D}$, conform with the system (1).

$$
\left\{\begin{array}{l}
\varphi_{2}=\varphi \pm \hat{B} \\
\varphi_{3}=\varphi \mp \hat{D}
\end{array}\right.
$$

First, one calculates the variable length 1 between B and D (system 2) [3-4].

$$
\left\{\begin{array}{l}
l^{2}=\left(x_{D}-x_{B}\right)^{2}+\left(y_{D}-y_{B}\right)^{2} \\
l=\sqrt{\left(x_{D}-x_{B}\right)^{2}+\left(y_{D}-y_{B}\right)^{2}}
\end{array}\right.
$$

The fi angle's parameters are determined with the system 3 [4].

$$
\left\{\begin{array}{l}
\sin \varphi=\frac{y_{D}-y_{B}}{l} \\
\cos \varphi=\frac{x_{D}-x_{B}}{l} \\
g \varphi=\frac{y_{D}-y_{B}}{x_{D}-x_{B}} \\
\varphi=\operatorname{sign}(\sin \varphi) \cdot \arccos (\cos \varphi)= \\
=\operatorname{sign}\left(\frac{y_{D}-y_{B}}{l}\right) \cdot \arccos \left(\frac{x_{D}-x_{B}}{l}\right)
\end{array}\right.
$$

The velocity of the angle fi is writing with the relationship (4), and the acceleration is determined with the system (5).

$$
\begin{aligned}
& \dot{\varphi}=\frac{\left(\dot{y}_{D}-\dot{y}_{B}\right) \cdot \cos \varphi-\left(\dot{x}_{D}-\dot{x}_{B}\right) \cdot \sin \varphi}{l}= \\
& =\frac{\left(\dot{y}_{D}-\dot{y}_{B}\right) \cdot\left(x_{D}-x_{B}\right)-\left(\dot{x}_{D}-\dot{x}_{B}\right) \cdot\left(y_{D}-y_{B}\right)}{l^{2}}= \\
& =\frac{\left(\dot{y}_{D}-\dot{y}_{B}\right) \cdot\left(x_{D}-x_{B}\right)-\left(\dot{x}_{D}-\dot{x}_{B}\right) \cdot\left(y_{D}-y_{B}\right)}{\left(x_{D}-x_{B}\right)^{2}+\left(y_{D}-y_{B}\right)^{2}}
\end{aligned}
$$

$i=\frac{\left(x_{D}-x_{B}\right) \cdot\left(\dot{x}_{D}-\dot{x}_{B}\right)+\left(y_{D}-y_{B}\right) \cdot\left(\dot{y}_{D}-\dot{y}_{B}\right)}{l}$

$\ddot{\varphi}=\frac{\left(\ddot{y}_{D}-\ddot{y}_{B}\right) \cdot \cos \varphi-\left(\ddot{x}_{D}-\ddot{x}_{B}\right) \cdot \sin \varphi-2 \cdot \dot{l} \cdot \dot{\varphi}}{l}$

Next, we will determine the kinematic parameters of the angle fi2 (system 6), and fi3 (system 7), from the triangle BCD [4]. 


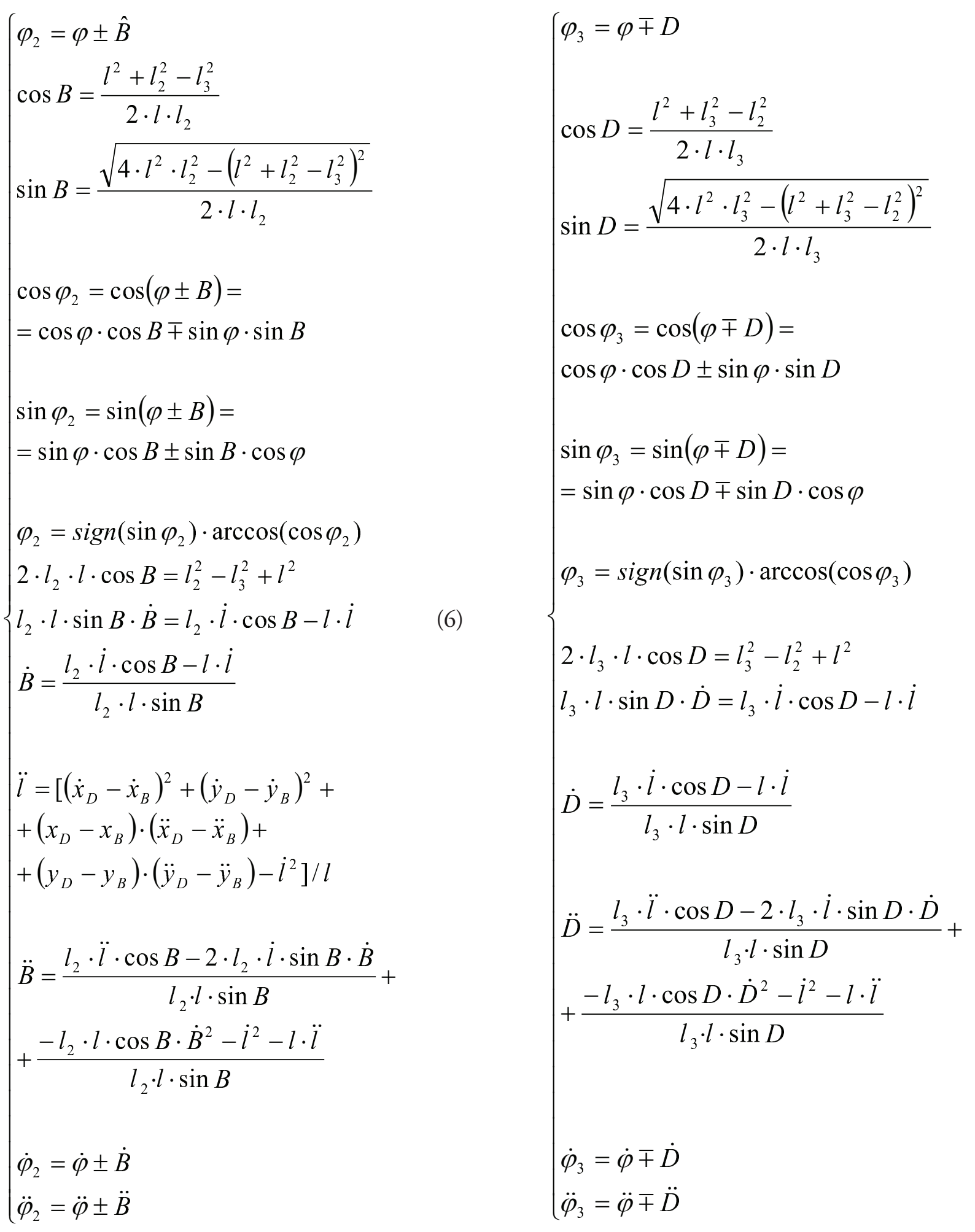


Finally, one determines the parameters of point C (system 8).

$$
\left\{\begin{array}{l}
x_{C}=x_{B}+l_{2} \cdot \cos \varphi_{2} \\
y_{C}=y_{B}+l_{2} \cdot \sin \varphi_{2} \\
\dot{x}_{C}=\dot{x}_{B}-l_{2} \cdot \sin \varphi_{2} \cdot \omega_{2} \\
\dot{y}_{C}=\dot{y}_{B}+l_{2} \cdot \cos \varphi_{2} \cdot \omega_{2} \\
\ddot{x}_{C}=\ddot{x}_{B}-l_{2} \cdot \cos \varphi_{2} \cdot \omega_{2}^{2}-l_{2} \cdot \sin \varphi_{2} \cdot \varepsilon_{2} \\
\ddot{y}_{C}=\ddot{y}_{B}-l_{2} \cdot \sin \varphi_{2} \cdot \omega_{2}^{2}+l_{2} \cdot \cos \varphi_{2} \cdot \varepsilon_{2}
\end{array}\right.
$$

\section{A combined method}

The kinematic schema of a RRR dyad can be seen in the Figure 1 [2-3].

The velocities can be determined faster using the vector method, so, to the second (proposed, combined) method, one uses the first way for the positions, and the vector method for the determining of the velocities and the accelerations [1-4].

The most difficult problem at the $3 \mathrm{R}$ dyad is the determining of the positions. To eliminate the traditional processes, to which we need two times to rises squared the system equations, we will determine the positions with the direct relationships (9) [4].

$$
\left\{\begin{array}{l}
\sin \varphi=\frac{y_{D}-y_{B}}{l} ; \\
\cos \varphi=\frac{x_{D}-x_{B}}{l} ; \\
\varphi=\operatorname{sign}(\sin \varphi) \cdot \arccos (\cos \varphi) \\
\cos B=\frac{l^{2}+l_{2}^{2}-l_{3}^{2}}{2 \cdot l \cdot l_{2}} \\
B=\arccos (\cos B) \\
\cos D=\frac{l^{2}+l_{3}^{2}-l_{2}^{2}}{2 \cdot l \cdot l_{3}} \\
D=\arccos (\cos D) \\
\varphi_{2}=\varphi \pm \hat{B} \\
\varphi_{3}=\varphi \mp \hat{D}
\end{array}\right.
$$

For the determination of velocities and accelerations one uses the classical vector method (systems 10-11) [4].

$$
\begin{aligned}
& \left\{\begin{array}{l}
\left\{\begin{array}{l}
l_{2} \cdot \cos \varphi_{2}+l_{3} \cdot \cos \varphi_{3}=x_{D}-x_{B} \\
l_{2} \cdot \sin \varphi_{2}+l_{3} \cdot \sin \varphi_{3}=y_{D}-y_{B}
\end{array}\right. \\
\left\{\begin{array}{l}
-l_{2} \cdot \sin \varphi_{2} \cdot \omega_{2}-l_{3} \cdot \sin \varphi_{3} \cdot \omega_{3}= \\
=\dot{x}_{D}-\dot{x}_{B} \\
l_{2} \cdot \cos \varphi_{2} \cdot \omega_{2}+l_{3} \cdot \cos \varphi_{3} \cdot \omega_{3}= \\
=\dot{y}_{D}-\dot{y}_{B}
\end{array}\right. \\
\left\{\begin{array}{l}
-l_{2} \cdot \sin \varphi_{2} \cdot \omega_{2}-l_{3} \cdot \sin \varphi_{3} \cdot \omega_{3}= \\
=\dot{x}_{D}-\dot{x}_{B} \mid \cdot\left(\cos \varphi_{3}\right) \\
l_{2} \cdot \cos \varphi_{2} \cdot \omega_{2}+l_{3} \cdot \cos \varphi_{3} \cdot \omega_{3}= \\
=\dot{y}_{D}-\dot{y}_{B} \mid \cdot\left(\sin \varphi_{3}\right)
\end{array}\right. \\
\Rightarrow \omega_{2}= \\
=\frac{\left(\dot{x}_{D}-\dot{x}_{B}\right) \cdot \cos \varphi_{3}+\left(\dot{y}_{D}-\dot{y}_{B}\right) \cdot \sin \varphi_{3}}{l_{2} \cdot \sin \left(\varphi_{3}-\varphi_{2}\right)} \\
\left\{\begin{array}{l}
-l_{2} \cdot \sin \varphi_{2} \cdot \omega_{2}-l_{3} \cdot \sin \varphi_{3} \cdot \omega_{3}= \\
=\dot{x}_{D}-\dot{x}_{B} \mid \cdot\left(\cos \varphi_{2}\right) \\
l_{2} \cdot \cos \varphi_{2} \cdot \omega_{2}+l_{3} \cdot \cos \varphi_{3} \cdot \omega_{3}= \\
=\dot{y}_{D}-\dot{y}_{B} \mid \cdot\left(\sin \varphi_{2}\right)
\end{array}\right. \\
\Rightarrow \omega_{3}= \\
=\frac{\left(\dot{x}_{D}-\dot{x}_{B}\right) \cdot \cos \varphi_{2}+\left(\dot{y}_{D}-\dot{y}_{B}\right) \cdot \sin \varphi_{2}}{l_{3} \cdot \sin \left(\varphi_{2}-\varphi_{3}\right)}
\end{array}\right. \\
& \int\left\{\begin{array}{l}
-l_{2} \cdot \sin \varphi_{2} \cdot \omega_{2}-l_{3} \cdot \sin \varphi_{3} \cdot \omega_{3}=\dot{x}_{D}-\dot{x}_{B} \\
l_{2} \cdot \cos \varphi_{2} \cdot \omega_{2}+l_{3} \cdot \cos \varphi_{3} \cdot \omega_{3}=\dot{y}_{D}-\dot{y}_{B}
\end{array} \Rightarrow\right. \\
& \left(-l_{2} \cdot \cos \varphi_{2} \cdot \omega_{2}^{2}-l_{2} \cdot \sin \varphi_{2} \cdot \varepsilon_{2}-\right. \\
& -l_{3} \cdot \cos \varphi_{3} \cdot \omega_{3}^{2}-l_{3} \cdot \sin \varphi_{3} \cdot \varepsilon_{3}=\ddot{x}_{D}-\ddot{x}_{B} \\
& -l_{2} \cdot \sin \varphi_{2} \cdot \omega_{2}^{2}+l_{2} \cdot \cos \varphi_{2} \cdot \varepsilon_{2}- \\
& -l_{3} \cdot \sin \varphi_{3} \cdot \omega_{3}^{2}+l_{3} \cdot \cos \varphi_{3} \cdot \varepsilon_{3}=\ddot{y}_{D}-\ddot{y}_{B} \\
& \left(-l_{2} \cdot \cos \varphi_{2} \cdot \omega_{2}^{2}-l_{2} \cdot \sin \varphi_{2} \cdot \varepsilon_{2}-l_{3} \cdot \cos \varphi_{3} \cdot \omega_{3}^{2}-\right. \\
& -l_{3} \cdot \sin \varphi_{3} \cdot \varepsilon_{3}=\ddot{x}_{D}-\ddot{x}_{B} \mid \cdot\left(\cos \varphi_{3}\right) \\
& -l_{2} \cdot \sin \varphi_{2} \cdot \omega_{2}^{2}+l_{2} \cdot \cos \varphi_{2} \cdot \varepsilon_{2}-l_{3} \cdot \sin \varphi_{3} \cdot \omega_{3}^{2}+ \\
& l_{3} \cdot \cos \varphi_{3} \cdot \varepsilon_{3}=\ddot{y}_{D}-\ddot{y}_{B} \mid \cdot\left(\sin \varphi_{3}\right) \\
& \left\{\varepsilon_{2}=\frac{\left(\ddot{x}_{D}-\ddot{x}_{B}\right) \cdot \cos \varphi_{3}+\left(\ddot{y}_{D}-\ddot{y}_{B}\right) \cdot \sin \varphi_{3}}{l_{2} \cdot \sin \left(\varphi_{3}-\varphi_{2}\right)}+\right. \\
& +\frac{l_{2} \cdot \omega_{2}^{2} \cdot \cos \left(\varphi_{3}-\varphi_{2}\right)+l_{3} \cdot \omega_{3}^{2}}{l_{2} \cdot \sin \left(\varphi_{3}-\varphi_{2}\right)} \\
& \left(-l_{2} \cdot \cos \varphi_{2} \cdot \omega_{2}^{2}-l_{2} \cdot \sin \varphi_{2} \cdot \varepsilon_{2}-l_{3} \cdot \cos \varphi_{3} \cdot \omega_{3}^{2}-\right. \\
& -l_{3} \cdot \sin \varphi_{3} \cdot \varepsilon_{3}=\ddot{x}_{D}-\ddot{x}_{B} \mid \cdot\left(\cos \varphi_{2}\right) \\
& -l_{2} \cdot \sin \varphi_{2} \cdot \omega_{2}^{2}+l_{2} \cdot \cos \varphi_{2} \cdot \varepsilon_{2}-l_{3} \cdot \sin \varphi_{3} \cdot \omega_{3}^{2}+ \\
& +l_{3} \cdot \cos \varphi_{3} \cdot \varepsilon_{3}=\ddot{y}_{D}-\ddot{y}_{B} \mid \cdot\left(\sin \varphi_{2}\right) \\
& \Rightarrow \varepsilon_{3}=\frac{\left(\ddot{x}_{D}-\ddot{x}_{B}\right) \cdot \cos \varphi_{2}+\left(\ddot{y}_{D}-\ddot{y}_{B}\right) \cdot \sin \varphi_{2}}{l_{3} \cdot \sin \left(\varphi_{2}-\varphi_{3}\right)}+ \\
& +\frac{l_{2} \cdot \omega_{2}^{2}+l_{3} \cdot \omega_{3}^{2} \cdot \cos \left(\varphi_{2}-\varphi_{3}\right)}{l_{3} \cdot \sin \left(\varphi_{2}-\varphi_{3}\right)}
\end{aligned}
$$




\section{A geometric method}

The kinematic schema of a RRR dyad can be seen in the Figure 1.

The third (proposed) method, is a geometric method, which determine first the kinematic parameters of the internal couple $(\mathrm{C})$ and then the rotation angles with their derivatives [1].

We start with the geometric positions (the system 12) [4].

$$
\left\{\begin{array}{l}
\left(x-x_{B}\right)^{2}+\left(y-y_{B}\right)^{2}=l_{2}^{2} \\
\left(x-x_{D}\right)^{2}+\left(y-y_{D}\right)^{2}=l_{3}^{2}
\end{array}\right.
$$

These equations were deduced geometrically, by the writing of two equations for a two circles $\left(\mathrm{x}=\mathrm{x}_{\mathrm{C}}, \mathrm{y}=\mathrm{y}_{\mathrm{C}}\right)$.

To solving the system (12), it writing the next relations (system 13) [4].

$$
\begin{aligned}
& \left\{\begin{array}{l}
\left(x-x_{B}\right)^{2}+\left(y-y_{B}\right)^{2}=l_{2}^{2} \\
\left(x-x_{D}\right)^{2}+\left(y-y_{D}\right)^{2}=l_{3}^{2}
\end{array}\right. \\
& \left(y-y_{B}\right)^{2}=l_{2}^{2}-\left(x-x_{B}\right)^{2} \\
& x-x_{D}= \pm \sqrt{l_{3}^{2}-\left(y-y_{D}\right)^{2}} \text {; } \\
& x=x_{D} \pm \sqrt{l_{3}^{2}-\left(y-y_{D}\right)^{2}} \text {; } \\
& x-x_{B}=\left(x_{D}-x_{B}\right) \pm \sqrt{l_{3}^{2}-\left(y-y_{D}\right)^{2}} \\
& \left(x-x_{B}\right)^{2}=\left(x_{D}-x_{B}\right)^{2}+\left[l_{3}^{2}-\left(y-y_{D}\right)^{2}\right] \pm \\
& \pm 2 \cdot\left(x_{D}-x_{B}\right) \cdot \sqrt{l_{3}^{2}-\left(y-y_{D}\right)^{2}} \\
& \left(x-x_{B}\right)^{2}=\left(x_{D}-x_{B}\right)^{2}+l_{3}^{2}-\left(y-y_{D}\right)^{2} \pm \\
& \pm 2 \cdot\left(x_{D}-x_{B}\right) \cdot \sqrt{l_{3}^{2}-\left(y-y_{D}\right)^{2}} \\
& \left(y-y_{B}\right)^{2}=l_{2}^{2}-\left(x_{D}-x_{B}\right)^{2}-l_{3}^{2}+ \\
& +\left(y-y_{D}\right)^{2} \mp 2 \cdot\left(x_{D}-x_{B}\right) \cdot \sqrt{l_{3}^{2}-\left(y-y_{D}\right)^{2}} \\
& y^{2}+y_{B}^{2}-2 \cdot y_{B} \cdot y=l_{2}^{2}-\left(x_{D}-x_{B}\right)^{2}- \\
& -l_{3}^{2}+y^{2}+y_{D}^{2}-2 \cdot y_{D} \cdot y \mp \\
& \mp 2 \cdot\left(x_{D}-x_{B}\right) \cdot \sqrt{l_{3}^{2}-\left(y-y_{D}\right)^{2}}
\end{aligned}
$$

$2 \cdot\left(y_{D}-y_{B}\right) \cdot y+$

$+\left[y_{B}^{2}-l_{2}^{2}+\left(x_{D}-x_{B}\right)^{2}+l_{3}^{2}-y_{D}^{2}\right]=$

$=\mp 2 \cdot\left(x_{D}-x_{B}\right) \cdot \sqrt{l_{3}^{2}-\left(y-y_{D}\right)^{2}}$

$2 \cdot\left(y_{D}-y_{B}\right)=b ;$

$y_{B}^{2}-l_{2}^{2}+\left(x_{D}-x_{B}\right)^{2}+l_{3}^{2}-y_{D}^{2}=d ;$

$2 \cdot\left(x_{D}-x_{B}\right)=a$

$b \cdot y+d=\mp a \cdot \sqrt{l_{3}^{2}-\left(y-y_{D}\right)^{2}}$

$b^{2} \cdot y^{2}+d^{2}+2 \cdot b \cdot d \cdot y=$

$=a^{2} \cdot l_{3}^{2}-a^{2} \cdot y^{2}-a^{2} \cdot y_{D}^{2}+2 \cdot a^{2} \cdot y_{D} \cdot y$

$\left(a^{2}+b^{2}\right) \cdot y^{2}-2 \cdot\left(a^{2} \cdot y_{D}-b \cdot d\right) \cdot y-$

$-\left(a^{2} \cdot l_{3}^{2}-a^{2} \cdot y_{D}^{2}-d^{2}\right)=0$

$\Delta(R)=\left(a^{2} \cdot y_{D}-b \cdot d\right)^{2}+$

$+\left(a^{2}+b^{2}\right) \cdot\left(a^{2} \cdot l_{3}^{2}-a^{2} \cdot y_{D}^{2}-d^{2}\right)=$

$=a^{4} \cdot y_{D}^{2}-a^{4} \cdot y_{D}^{2}+b^{2} \cdot d^{2}-b^{2} \cdot d^{2}-$

$-2 \cdot a^{2} \cdot b \cdot d \cdot y_{D}-a^{2} \cdot d^{2}+$

$+a^{4} \cdot l_{3}^{2}+a^{2} \cdot b^{2} \cdot l_{3}^{2}-a^{2} \cdot b^{2} \cdot y_{D}^{2}=$

$=a^{2} \cdot\left[l_{3}^{2} \cdot\left(a^{2}+b^{2}\right)-\left(d+b \cdot y_{D}\right)^{2}\right]$

$c=x_{B}^{2}-x_{D}^{2}+y_{B}^{2}-y_{D}^{2}+l_{3}^{2}-l_{2}^{2}$

$y_{1,2}=\frac{a^{2} \cdot y_{D}-b \cdot d \pm a \cdot \sqrt{l_{3}^{2} \cdot\left(a^{2}+b^{2}\right)-\left(d+b \cdot y_{D}\right)^{2}}}{a^{2}+b^{2}} ;$

$x_{1,2}=-\frac{b}{a} \cdot y_{1,2}-\frac{c}{a}$

+when $C$ a North - when $C$ a South

$y_{1,2}=\frac{a^{2} \cdot y_{D}-b \cdot d \pm a \cdot \sqrt{l_{3}^{2} \cdot\left(a^{2}+b^{2}\right)-\left(d+b \cdot y_{D}\right)^{2}}}{a^{2}+b^{2}} ;$ 
To determine the velocities and the accelerations we derived the equations system (12) and we have obtained the relations (system 14) [4].

$$
\begin{aligned}
& 2 \cdot\left(x-x_{B}\right) \cdot\left(\dot{x}-\dot{x}_{B}\right)+ \\
& +2 \cdot\left(y-y_{B}\right) \cdot\left(\dot{y}-\dot{y}_{B}\right)=0 \\
& 2 \cdot\left(x-x_{D}\right) \cdot\left(\dot{x}-\dot{x}_{D}\right)+ \\
& +2 \cdot\left(y-y_{D}\right) \cdot\left(\dot{y}-\dot{y}_{D}\right)=0 \\
& \left(x-x_{B}\right) \cdot \dot{x}+\left(y-y_{B}\right) \cdot \dot{y}= \\
& =\left(x-x_{B}\right) \cdot \dot{x}_{B}+\left(y-y_{B}\right) \cdot \dot{y}_{B} \\
& \left(x-x_{D}\right) \cdot \dot{x}+\left(y-y_{D}\right) \cdot \dot{y}= \\
& =\left(x-x_{D}\right) \cdot \dot{x}_{D}+\left(y-y_{D}\right) \cdot \dot{y}_{D}
\end{aligned}
$$

$$
a_{\mathbb{1}}=x-x_{B} ; \quad a_{\mathrm{2}}=y-y_{B} ;
$$$$
b_{1}=\left(x-x_{B}\right) \cdot \dot{x}_{B}+\left(y-y_{B}\right) \cdot \dot{y}_{B}
$$$$
a_{2}=x-x_{D} ; a_{2}=y-y_{D} \text {; }
$$$$
b_{2}=\left(x-x_{D}\right) \cdot \dot{x}_{D}+\left(y-y_{D}\right) \cdot \dot{y}_{D}
$$$$
\Delta=\left|\begin{array}{ll}
a_{1} & a_{\mathrm{P}} \\
a_{1} & a_{2}
\end{array}\right|=a_{1} \cdot a_{2}-a_{1} \cdot a_{\mathrm{P}} ;
$$$$
\Delta_{\dot{x}}=\left|\begin{array}{ll}
b_{1} & a_{\mathrm{P}} \\
b_{2} & a_{2}
\end{array}\right|=b_{1} \cdot a_{2}-b_{2} \cdot a_{\mathrm{P}} \text {; }
$$$$
\Delta_{\dot{y}}=\left|\begin{array}{ll}
a_{1} & b_{1} \\
a_{2} & b_{2}
\end{array}\right|=a_{1} \cdot b_{2}-a_{2} \cdot b_{1} ;
$$$$
\dot{x} \equiv \dot{x}_{C}=\frac{\Delta \dot{x}}{\Delta} ; \quad \dot{y} \equiv \dot{y}_{C}=\frac{\Delta \dot{y}}{\Delta}
$$$$
\left(\dot{x}-\dot{x}_{B}\right) \cdot \dot{x}+\left(x-x_{B}\right) \cdot \ddot{x}+
$$$$
+\left(\dot{y}-\dot{y}_{B}\right) \cdot \dot{y}+\left(y-y_{B}\right) \cdot \ddot{y}=
$$$$
=\left(\dot{x}-\dot{x}_{B}\right) \cdot \dot{x}_{B}+\left(x-x_{B}\right) \cdot \ddot{x}_{B}+
$$$$
+\left(\dot{y}-\dot{y}_{B}\right) \cdot \dot{y}_{B}+\left(y-y_{B}\right) \cdot \ddot{y}_{B}
$$

$$
\begin{aligned}
& \left(\dot{x}-\dot{x}_{D}\right) \cdot \dot{x}+\left(x-x_{D}\right) \cdot \ddot{x}+ \\
& +\left(\dot{y}-\dot{y}_{D}\right) \cdot \dot{y}+\left(y-y_{D}\right) \cdot \ddot{y}= \\
& =\left(\dot{x}-\dot{x}_{D}\right) \cdot \dot{x}_{D}+\left(x-x_{D}\right) \cdot \ddot{x}_{D}+ \\
& +\left(\dot{y}-\dot{y}_{D}\right) \cdot \dot{y}_{D}+\left(y-y_{D}\right) \cdot \ddot{y}_{D} \\
& \left\{\begin{array}{l}
a_{1} \cdot \ddot{x}+a_{1} \cdot \ddot{y}=c_{1} \\
a_{2} \cdot \ddot{x}+a_{2} \cdot \ddot{y}=c_{2}
\end{array}\right. \\
& \left\{\begin{array}{l}
c_{1}=\left(x-x_{B}\right) \cdot \ddot{x}_{B}+\left(y-y_{B}\right) \cdot \ddot{y}_{B}- \\
-\left(\dot{x}-\dot{x}_{B}\right)^{2}-\left(\dot{y}-\dot{y}_{B}\right)^{2} \\
c_{2}=\left(x-x_{D}\right) \cdot \ddot{x}_{D}+\left(y-y_{D}\right) \cdot \ddot{y}_{D}- \\
-\left(\dot{x}-\dot{x}_{D}\right)^{2}-\left(\dot{y}-\dot{y}_{D}\right)^{2}
\end{array}\right. \\
& \Delta_{\ddot{x}}=\left|\begin{array}{ll}
c_{1} & a_{\mathfrak{1}} \\
c_{2} & a_{2}
\end{array}\right|=a_{2} \cdot c_{1}-a_{1} \cdot c_{2} ; \\
& \ddot{x} \equiv \ddot{x}_{C}=\frac{\Delta \ddot{x}}{\Delta} ; \\
& \Delta_{\ddot{y}}=\left|\begin{array}{ll}
a_{1} & c_{1} \\
a_{2} & c_{2}
\end{array}\right|=a_{1} \cdot c_{2}-a_{1} \cdot c_{1} ; \\
& \ddot{y} \equiv \ddot{y}_{C}=\frac{\Delta \ddot{y}}{\Delta}
\end{aligned}
$$

Finally one determines the positions angles and their derivatives (system 15) [4].

$$
\begin{aligned}
& \left\{\begin{array}{l}
x_{C}=x_{B}+l_{2} \cdot \cos \varphi_{2} \\
y_{C}=y_{B}+l_{2} \cdot \sin \varphi_{2}
\end{array}\right. \\
& \left\{\begin{array}{l}
x_{D}=x_{C}+l_{3} \cdot \cos \varphi_{3} \\
y_{D}=y_{C}+l_{3} \cdot \sin \varphi_{3}
\end{array}\right. \\
& \left\{\begin{array}{l}
x_{C}-x_{B}=l_{2} \cdot \cos \varphi_{2} \\
y_{C}-y_{B}=l_{2} \cdot \sin \varphi_{2}
\end{array}\right. \\
& \left\{\begin{array}{l}
x_{D}-x_{C}=l_{3} \cdot \cos \varphi_{3} \\
y_{D}-y_{C}=l_{3} \cdot \sin \varphi_{3}
\end{array}\right. \\
& \cos \varphi_{2}=\frac{x_{C}-x_{B}}{l_{2}} ; \sin \varphi_{2}=\frac{y_{C}-y_{B}}{l_{2}} ; \\
& \cos \varphi_{3}=\frac{x_{D}-x_{C}}{l_{3}} ; \sin \varphi_{3}=\frac{y_{D}-y_{C}}{l_{3}}
\end{aligned}
$$


$\left\{\begin{array}{l}\dot{x}_{C}-\dot{x}_{B}=-l_{2} \cdot \sin \varphi_{2} \cdot \omega_{2} \mid \cdot\left(-\sin \varphi_{2}\right) \\ \dot{y}_{C}-\dot{y}_{B}=l_{2} \cdot \cos \varphi_{2} \cdot \omega_{2} \mid \cdot\left(\cos \varphi_{2}\right)\end{array} \Rightarrow\right.$

$\Rightarrow \omega_{2}=\frac{\left(\dot{y}_{C}-\dot{y}_{B}\right) \cdot \cos \varphi_{2}-\left(\dot{x}_{C}-\dot{x}_{B}\right) \cdot \sin \varphi_{2}}{l_{2}}$

$\left\{\begin{array}{l}\dot{x}_{D}-\dot{x}_{C}=-l_{3} \cdot \sin \varphi_{3} \cdot \omega_{3} \mid \cdot\left(-\sin \varphi_{3}\right) \\ \dot{y}_{D}-\dot{y}_{C}=l_{3} \cdot \cos \varphi_{3} \cdot \omega_{3} \mid \cdot\left(\cos \varphi_{3}\right)\end{array} \Rightarrow\right.$

$\Rightarrow \omega_{3}=\frac{\left(\dot{y}_{D}-\dot{y}_{C}\right) \cdot \cos \varphi_{3}-\left(\dot{x}_{D}-\dot{x}_{C}\right) \cdot \sin \varphi_{3}}{l_{3}}$

$\left\{\begin{array}{l}\ddot{x}_{C}-\ddot{x}_{B}=-l_{2} \cos \varphi_{2} \cdot \omega_{2}^{2}-l_{2} \sin \varphi_{2} \cdot \varepsilon_{2} \mid-\sin \varphi_{2} \\ \ddot{y}_{C}-\ddot{y}_{B}=-l_{2} \sin \varphi_{2} \cdot \omega_{2}^{2}+l_{2} \cos \varphi_{2} \cdot \varepsilon_{2} \mid \cos \varphi_{2}\end{array} \Rightarrow\right.$

$\Rightarrow \varepsilon_{2}=\frac{\left(\ddot{y}_{C}-\ddot{y}_{B}\right) \cdot \cos \varphi_{2}-\left(\ddot{x}_{C}-\ddot{x}_{B}\right) \cdot \sin \varphi_{2}}{l_{2}}$

$\left\{\begin{array}{l}\ddot{x}_{D}-\ddot{x}_{C}=-l_{3} \cos \varphi_{3} \cdot \omega_{3}^{2}-l_{3} \sin \varphi_{3} \cdot \varepsilon_{3} \mid-\sin \varphi_{3} \\ \ddot{y}_{D}-\ddot{y}_{C}=-l_{3} \sin \varphi_{3} \cdot \omega_{3}^{2}+l_{3} \cos \varphi_{3} \cdot \varepsilon_{3} \mid \cos \varphi_{3}\end{array} \Rightarrow\right.$

$\Rightarrow \varepsilon_{3}=\frac{\left(\ddot{y}_{D}-\ddot{y}_{C}\right) \cdot \cos \varphi_{3}-\left(\ddot{x}_{D}-\ddot{x}_{C}\right) \cdot \sin \varphi_{3}}{l_{3}}$

\section{Conclusions}

The geometrical method presented in the last paragraph is the most elegant and direct method to determine the positions angles and their derivatives [4].

The relationships presented in this paper allow and the synthesis of robots (the mechanical systems, serial, in movement) [4].

\section{References}

[1] Comanescu, A., ş.a., Bazele modelarii mecanismelor. E.Politeh. Press, Bucureşti, 2010, ISBN 13: 978-606515-114-7, page count 274.

[2] Erdman, A.G., Three and four precision point kinematic synthesis of planar linkages, Elsevier, Mechanism and Machine Theory, Volume 16, Issue 3, 1981, pages 227-245.

[3] Gibson, C.G., Newstead, P.E., On the geometry of the planar 4-bar mechanism, Acta Applicandae Mathematicae, Volume 7, Number 2, pages 113-135.

[4] Petrescu, F.I., Teoria Mecanismelor si a Masinilor, Create Space Publisher, USA, 2011, ISBN/EAN 13: 1468015826 / 978-1-4680-1582-9, page count 432. 